ARTIGO DE ATUALIZAÇÃO

\title{
FÍSTULAS DIGESTIVAS NO TRAUMA
}

\section{DIGESTIVE FISTULAS IN TRAUMA}

\section{Hamilton Petry de Souza, TCBC-RS ${ }^{1}$ \\ Gémerson Gabiatti² Fernanda Dotta ${ }^{3}$}

\section{INTRODUÇÃO}

Fístulas são comunicações anormais entre duas superfícies epitelizadas e o seu surgimento representa uma séria complicação. Em função disso, há poucos problemas cirúrgicos que necessitem mais atenção do que uma fístula digestiva (FD). Até $85 \%$ ocorrem como complicações pósoperatórias, especialmente em situações de urgência. Acontecem normalmente entre o quinto e o décimo dia pós-operatório (PO), em função de problemas na linha de sutura como tensão excessiva, má vascularização e técnica inadequada. Qualquer circunstância em que haja um defeito na parede do órgão ou crie condição que interfira com a cicatrização normal pode levar à formação de uma FD. Alguns dos fatores implicados podem ser controlados pelo cirurgião, como o preparo pré-operatório adequado, uso correto de antibióticos, técnica cirúrgica meticulosa, fechamento seguro da parede e manutenção de transporte adequado de oxigênio no $\mathrm{PO}^{1,2}$.

Os autores fazem uma revisão acerca das fístulas digestivas com ênfase nas complicações pós-trauma, destacando classificação, fisiopatologia, complicações, métodos diagnósticos e tratamento. $\mathrm{O}$ tema será abordado estratificando-o em relação ao órgão fistuloso.

\section{CLASSIFICAÇÃO}

As FDs podem ser classificadas do ponto de vista anatômico, fisiológico e etiológico ${ }^{1}$.

1) Anatômico: Podem ser divididas em internas (comunicações anômalas entre órgãos intracavitários, p.ex., fístula colecistoentérica) e externas (entre um órgão intracavitário e a superfície cutânea, p.ex., fístula colotocutânea). A Tabela 1 inclui detalhes anatômicos e seus significados quanto à evolução.

2) Fisiológico: $\mathrm{O}$ volume do efluente em 24 horas classifica as FDs em alto (maior que $500 \mathrm{ml}$ ), moderado (entre 200 e $500 \mathrm{ml}$ ) e baixo (menor que $200 \mathrm{ml}$ ) débitos. Esses valores são importantes ao se considerar o tratamento, uma vez que o débito fistuloso, além de produzir importante impacto fisiológico é fator prognóstico independente de probabilidade de fechamento e de óbito.

3) Etiológico: Refere-se ao processo que determinou o surgimento do processo fistuloso, p.ex., apendicite, diverticulite e trauma.

\section{DIAGNÓSTICO E TRATAMENTO}

O manejo das FDs obedece a etapas que se iniciam no reconhecimento de fatores que possam determinar seu surgimento como o tipo de operação, lesões existentes, correção efetuada, sendo a atenção aos aspectos técnicos fundamental na sua "prevenção". Além desses, há fatores como presença de corpo estranho, radioterapia prévia, doenças inflamatórias intestinais, epitelização do trajeto, neoplasia associada e obstrução distal que podem prejudicar seu fechamento ${ }^{3,4}$. Devem ser controladas as complicações mais ameaçadoras das FDs como o distúrbio hidreletrolítico, sepse e desnutrição. Para tal, hidratação adequada, uso criterioso de antibióticos, correção da anemia, drenagem da coleção ou abscesso, suporte nutricional e controle do débito fistuloso são indicados ${ }^{5}$. O uso do jejum absoluto e do

1. Coordenador da Residência em Cirurgia do Trauma do Hospital de Pronto Socorro de Porto Alegre. Professor Adjunto da Disciplina de Cirurgia Abdominal e Coordenador do Departamento de Cirurgia da Faculdade de Medicina da Pontifícia Universidade Católica do Rio Grande do Sul (FAMED-PUCRS). Chefe do Serviço de Cirurgia Geral do Hospital São Lucas (HSL)-PUCRS. Fellow American College of Surgeons - FACS.

2. Cirurgião Geral. Residente de Cirurgia do Trauma do HPS-POA.

3. Doutoranda da FAMED-PUCRS.

Recebido em 14/2/2000

Aceito para publicação em 1/8/2000 
Tabela 1

Características Anatômicas e Significado ${ }^{1}$

\begin{tabular}{l|l}
\hline Favorável & Desfavorável \\
\hline Continuidade visceral mantida & Destruição completa \\
Fístula terminal & Fístula lateral \\
Sem abscesso & Abscesso associado \\
Intestino adjacente saudável & Intestino adjacente \\
& patológico \\
Livre fluxo distal & Obstrução distal \\
Orofaringe, esôfago & Gástrico \\
Coto duodenal, pancratobiliar & Duodenal lateral \\
Jejunal & Ileal \\
Trato fistuloso $>2 \mathrm{~cm}$ & Trato fistuloso $<2 \mathrm{~cm}$ \\
Defeito visceral $<1 \mathrm{~cm}$ & Defeito visceral $>1 \mathrm{~cm}$ \\
\hline
\end{tabular}

tubo nasogástrico pode remover de 4 a 7 litros de secreção, sendo úteis em fístulas proximais. A investigação deve ser guiada conforme o sítio fistuloso em questão (fistulografia, tomografia computadorizada (TC), endoscopia). Uma vez diagnosticada a FD e controladas suas manifestações locais e sistêmicas, deve ser definida a opção de tratamento inicial, em princípio com a possibilidade de ocorrer fechamento espontâneo, levando-se em consideração detalhes anatômicos e fisiológicos. As perdas hidreletrolíticas, a desnutrição e a sepse provocam importante morbi-mortalidade. Em geral, a mortalidade das FDs situa-se entre 6,5 e $21 \%$ e a sepse é a complicação mais comum nas fístulas enterocutâneas ${ }^{6}$.

Nos últimos anos, a TC e a ultra-sonografia (US) demonstraram grande valor no diagnóstico de lesões intraabdominais e o seu papel terapêutico relacionado à drenagem percutânea (DP) tem sido realçado ${ }^{7}$. A DP foi inicialmente aplicada em coleções simples, sem uma FD conhecida associada. Percebeu-se posteriormente que muitas dessas estavam associadas a lesões fistulosas e que sua maioria fechava com a DP. Estima-se que a incidência de abscessos intra-abominais relacionados às FDs varie de 15 a $44 \%$ e que a maioria desses (até $84 \%$ ) poderia ser tratada via percutânea. Pacientes com focos infecciosos sem controle têm seu processo cicatricial prejudicado. Indicase o procedimento percutâneo em coleções associadas a fístulas de baixo débito, praticamente em todo trato digestivo. O material obtido deve ser enviado para análise microbiológica, a fim de auxiliar a escolha antibiótica. São descritas taxas de fechamento que variam de 50 a $84 \%$, ocorrendo a maioria em quatro a oito semanas ${ }^{8}$. Destacase que atualmente a TC é o método diagnóstico de escolha no diagnóstico de coleções peritoneais ${ }^{9,10}$. Complementarmente, os estudos radiológicos contrastados e cintilográficos podem ser úteis. Como meio de contraste, o bário é superior aos agentes hidrossolúveis por apresentar uma melhor definição anatômica das estruturas, embora tenha potencial irritativo importante se extravasar para uma superfície como o peritônio ou a pleura ${ }^{11}$.

As manifestações clínicas que devem alertar o cirurgião para a possibilidade de uma coleção intra-abdominal incluem dor, febre, calafrios, taquicardia, íleo prolongado, náuseas e vômitos, especialmente no período crítico para deiscências. Deve ser lembrado que infecções de parede abdominal podem ser fonte toxêmica importante, podendo culminar em infecção grave de partes moles (fasceíte, miosite, celulite), principalmente associadas a lesões gástricas, duodenais e jejunais proximais 2,4 .

A sepse e suas repercussões sistêmicas podem ser a manifestação inicial no paciente com fístula digestiva associada ou não a uma coleção intra-abdominal. A re-intervenção nesses pacientes é acompanhada de elevada morbimortalidade, devendo-se considerar essa opção somente após descartar outras alternativas menos invasivas. O paciente deve estar otimizado hemodinamicamente e as reservas de sangue e derivados adequadas. Idealmente devese definir a localização do foco no pré-operatório. A incisão deve ser guiada pela natureza e localização da infecção, presença de operações prévias e pela própria experiência do cirurgião. $\mathrm{O}$ ato de reoperar o abdome de pacientes com fístulas é um desafio ${ }^{2,12}$.

Os cuidados com o orifício fistuloso englobam três objetivos: controlar as perdas e estimar a reposição de volume e eletrólitos, proteção da pele e avaliação da evolução do tratamento empregado. A inserção de um cateter plástico maleável no orifício fistuloso permite um controle preciso do volume de drenagem, devendo ser trocado por um cateter progressivamente mais fino conforme ocorrer a cicatrização do trajeto, sendo então substituído por uma bolsa de ostomia. Os cuidados com a pele incluem a aplicação de óxido de zinco, pasta de alumínio ou dispositivos especiais como bolsa de Karaya e similares a fim de impedir o contato da pele com as secreções fistulosas ${ }^{4}$.

\section{Suporte Nutricional no Manejo das FDs}

A partir do trabalho de Chapman ${ }^{13}$ na década de 1960 reconheceu-se que o suporte nutricional adequado é parte essencial no tratamento das fístulas digestivas. Nesse estudo, as taxas de fechamento espontâneo e mortalidade foram de 89 e $12 \%$, comparadas a 37 e $55 \%$, respectivamente, com suporte nutricional inadequado. Deve ser iniciado precocemente, tendo como objetivo impedir maior deterioração do estado nutricional ${ }^{14}$.

A decisão quanto à via de administração, se parenteral ou enteral, deve ser feita em função do local da fístula. $\mathrm{O}$ advento da nutrição parenteral revolucionou o tratamento das fístulas. Em geral, é reservada para aquelas situações em que o trato digestivo não está "disponível" ou então se alterações morfofuncionais impedem sua utilização ${ }^{15,16,17}$. A via enteral, sempre que possível, deve ser a preferida pelo seu baixo custo, ampla disponibilidade, facilidade no manejo e pelo efeito trófico sobre a mucosa intestinal diminuindo a translocação bacteriana. $\mathrm{O}$ acesso enteral (cateter nasoentérico, jejuno ou gastrostomia) deveria ser providenciado em toda operação caso essa possa vir a apresentar como complicação uma FD ${ }^{4,14}$.

Os cálculos da necessidade calórica podem ser feitos através da equação de Harris-Benedict obtendo-se dessa forma o gasto energético basal (GEB), que deve ser multiplicado por um fator de estresse, que variará em função 
das condições gerais de cada paciente. Adiciona-se ao preparado eletrólitos e oligoelementos em suas doses habituais. O tempo de duração do suporte nutricional irá variar conforme a evolução do paciente ${ }^{14,18}$.

\section{Fatores Prognósticos nas FDs}

A mortalidade em intervenções eletivas em geral é de $2 \%$, enquanto nos pacientes com fístulas varia de 6 a $48 \%$. Essa grande variação nos dados reflete a heterogeneidade dos pacientes e suas fístulas. As comparações de mortalidade são difíceis, pois muitas variáveis influenciam os resultados. São exemplos, a origem da fístula, débito, se é interna ou externa, o grau de desnutrição associado, sepse local ou sistêmica, a resposta do paciente e doenças associadas ${ }^{3}$.

As características clínicas influenciam na decisão do tipo de tratamento que será empregado. Por isso a compreensão dos fatores que afetam o prognóstico em relação à possibilidade de fechamento espontâneo ou necessidade de intervenção cirúrgica é de grande valor ${ }^{3}$. A Tabela 2 resume alguns desses fatores e sua significância em relação à possibilidade de fechamento espontâneo e à mortalidade.

O fechamento espontâneo é mais frequiente nas fístulas bileopancreáticas e menos comum nas duodenojejunais. É também mais comum nas lesões distais do que nas proximais. A mortalidade também é maior nas fístulas proximais (em função do débito elevado). As complicações podem ser sépticas ou não. No passado os distúrbios hidreletrolíticos foram a maior causa de mortalidade. $\mathrm{O}$ controle de eletrólitos em fístulas de alto débito previne tais complicações. Atualmente a sepse é responsável por $80 \%$ dos óbitos ${ }^{3}$.

Fístulas com muitos orifícios de saída complicam o manejo da pele perilesional, podendo produzir grandes defeitos na parede abdominal. Essas lesões têm um potencial de fechamento espontâneo baixo. O mesmo princípio se aplica quando existirem múltiplos tratos fistulosos oriundos de vários órgãos ${ }^{4}$. A redução do débito fistuloso é uma das metas do tratamento, pois diminui as perdas nutricionais e hidreletrolíticas diminuindo a mortalidade e aumentando as taxas de fechamento. Edmunds descreveu sobre a relação do volume do efluente com a mortalidade. Pacientes com baixo e alto débito tinham taxas de óbito respectivas de 16 e $54 \%{ }^{19}$. O uso da nutrição parenteral e da somatostatina e seus análogos sintéticos vem a acrescentar com esse mesmo objetivo. A via oral pode, eventualmente, ser utilizada em fístulas de baixo débito, biliares e colônicas. Quanto às proteínas séricas sabe-se que a concentração de albumina tem importância preditiva em relação à mortalidade e ao fechamento. Quando essa for menor do que $2,5 \mathrm{~g} / \mathrm{dl}$ a mortalidade é de $42 \%$, comparada a zero se maior que $3,5 \mathrm{~g} / \mathrm{dl}$. A transferrina também pode ser utilizada com esse fim, com boa influência prognóstica associada a concentrações séricas maiores de $200 \mathrm{mg} / \mathrm{dl}^{1}$.

\section{Fístulas Esofágicas}

Devem ser divididas em cervicais, torácicas e abdominais. As fístulas cervicais ocorrem principalmente no PO devido à deiscência anastomótica, geralmente entre o $5^{\circ}$ e o $10^{\circ}$ dias. Tanto o trauma penetrante como o contuso podem determinar perfurações esofagianas, ocorrendo em $5,5 \%$ e $0,1 \%$, respectivamente ${ }^{20}$. A apresentação clínica inclui odinofagia, disfagia, edema, febre, leucocitose, dor e crepitação local em função do enfisema associado. Quando a perfuração está localizada abaixo do diafragma, a dor é vaga e principalmente confinada ao epigástrio ${ }^{21}$.O diag-

Tabela 2

Fatores prognósticos e significado quanto ao fechamento espontâneo e mortalidade ${ }^{3}$

\begin{tabular}{llll}
\hline Fator & Favorável & Desfavorável \\
\hline Características da FD & Trato longo & Trato curto \\
& Continuidade intestinal mantida & Eversão de mucosa \\
& Ausência de obstrução & Intestino adjacente patológico \\
& & Evisceração, íleo \\
& & Obstrução distal \\
& & Defeito da parede abdominal \\
Órgão de origem & Bileopancreático, colon & Gástrica, duodenal, jejunoileal \\
Sepse & Ausente & Presente \\
Etiologia & Deiscência anastomótica & Neoplasia, doença infamatória, radioterapia \\
& Doença inflamatória* & Deiscência anastomótica* \\
Idade & Sem influência & Sem influência \\
& $<50$ anos* & $>50$ anos* \\
Origem do paciente & Do próprio hospital & Paciente transferido \\
Débito & $<500 \mathrm{ml} /$ dia & $>500 \mathrm{ml} /$ dia \\
Desnutrição & Ausente & Presente \\
Tempo de evolução & Aguda & Crônica \\
& Crônica* & Aguda*
\end{tabular}


nóstico pode ser feito através do radiograma de tórax e estudos radiológicos contrastados do esôfago que podem revelar extravasamento de contraste, edema pré-vertebral, enfisema cervical, alargamento mediastinal, pneumomediastino, derrame pleural e pneumotórax ${ }^{6}$. A TC é o exame que define com precisão a lesão esofágica ${ }^{22}$. A endoscopia digestiva está indicada frente à suspeita de corpo estranho, no caso de lesão associada da via aérea ou quando existe forte suspeita de lesão, mas os exames contrastados são normais ${ }^{23}$.

As fístulas do esôfago torácico podem ser verdadeiras emergências em função da elevada incidência de sepse e disfunção de múltiplos órgãos. Sua etiologia inclui a instrumentação, os corpos estranhos, o trauma direto (p.ex. penetrante), nas lesões da transição tóraco-abdominal e transfixantes do mediastino e as operações sobre o órgão. Exigem um alto índice de suspeição ${ }^{6,21,22}$.

O tratamento da perfuração pós-instrumentação pode ser conservador desde que o paciente apresente um quadro clínico com evolução prolongada e branda (sem sepse associada) e uma perfuração bloqueada for demonstrada nos estudos radiológicos. Esse inclui antibióticos apropriados, jejum e exames seriados clínicos e laboratoriais. Havendo qualquer sinal de sepse local pode ocorrer extensão para o mediastino e suas temíveis consequiências, devendo o paciente ser submetido ao tratamento convencional. Se os estudos demonstrarem extravasamento grosseiro, celulite extensa ou abscesso, deve-se realizar drenagem cervical, com ou sem reparo da lesão esofágica ${ }^{2,22}$.

A maioria das fístulas cervicais pós-anastomóticas fecha espontaneamente em duas a três semanas, devendo seu tratamento, em princípio, ser conservador. Naquela situação em que existir grande destruição anastomótica ou sepse, o tratamento deve ser a drenagem cirúrgica da região cervical com ou sem o reparo da lesão, podendose utilizar a esofagostomia cervical. As lesões toracoabdominais devem ser tratadas com exclusão do trânsito e drenagem ampla. Quando submetidas à intervenção precoce pode-se optar por debridamento, sutura da lesão e cobertura por retalho (pleura, músculo intercostal, estômago ou omento) dependendo do sítio da lesão e das condições locais. $\mathrm{O}$ acesso enteral deve ser realizado nessa situação $6,20,22$.

\section{Fístulas Gastroduodenais}

A deiscência anastomótica é sua principal causadora (em torno de 95\% dos casos). O trauma responde por 5\% dos casos e a mortalidade média é de $23,5 \%$. A maioria se apresenta com dor abdominal, febre, leucocitose, drenagem purulenta ou entérica pela ferida operatória. O diagnóstico pode ser feito através de estudo radiológico contrastado, fistulografia ou TC. Esses estudos devem verificar qual a causa de fístula, a extensão da lesão, se há coleção associada ou obstrução distal ${ }^{24}$.

Essas lesões podem provocar grandes distúrbios hidreletrolíticos, desnutrição, e infecção e têm seus distúrbios agravados quanto maior for a proximidade com o ângu- lo de Treitz. O déficit causado é diretamente proporcional ao volume e composição do efluente. Cuidados com a proteção da pele, controle rigoroso dos sinais vitais, do balanço hídrico e dos exames laboratoriais são fundamentais. A hiponatremia, hipocalcemia, acidose metabólica e anemia são distúrbios comuns.

Nas lesões gástricas de alto débito pode ocorrer a alcalose metabólica. O uso da albumina exógena com o objetivo de elevar a pressão oncótica pode ser benéfico, embora o suporte nutricional seja crítico nessa situação. $O$ suporte nutricional precoce com a nutrição parenteral total (NPT) diminui o débito embora não produza impacto nas taxas de mortalidade e de fechamento espontâneo. A utilização de análogos da somatostatina parece diminuir o débito, mas não altera o índice de fechamento, embora, acredite-se que o tempo necessário para tal diminua com seu uso ${ }^{6}$.

Quanto aos fatores prognósticos envolvidos no manejo dessas lesões sabe-se que $90 \%$ das fístulas fecham espontaneamente em quatro a seis semanas. Como regra geral, caso não ocorra fechamento em seis semanas, é improvável que ocorra sem reparo cirúrgico. As taxas de fechamento, morbidade e mortalidade estão associadas com o tipo de fístula, número e a significância de erros no manejo, que podem ser precoces (falha no diagnóstico) ou tardios (falha em exteriorizar, em iniciar o suporte nutricional, demora na exploração cirúrgica e tentativa prematura em restaurar a continuidade do trato intestinal). O sítio da fístula pode ser importante no que tange ao potencial de fechamento, pois é sabido que as lesões duodenais fecham mais que as gástricas. $O$ débito elevado implica mortalidade de 38 a $45 \%$ em relação a quase zero nas lesões com baixo débito. A morfologia do trato fistuloso influencia no fechamento, uma vez que a presença de múltiplos trajetos tem mortalidade associada de $36 \%$ versus $25 \%$ nos trajetos únicos. A propensão para fechamento também é menor quando o trajeto é menor que $2 \mathrm{~cm}$. A presença de neoplasia local, a radioterapia, a presença de corpos estranhos, a obstrução distal e a epitelização do trajeto também afetam adversamente o fechamento das fístulas ${ }^{24}$.

As opções cirúrgicas incluem exclusão pilórica com gastroenterostomia e ressecção com diverticulização, entre outras. A exclusão pilórica e a diverticulização duodenal são opções de exclusão do trânsito, convertendo-se uma fístula fora de controle em uma fístula controlada ${ }^{25,26}$. Fístulas gástricas isoladas são raras e ocorrem principalmente no $\mathrm{PO}$ devido à deiscência anastomótica ou à isquemia parietal em função de ligaduras vasculares, p.ex., após esplenectomia. Os princípios gerais de manejo das FDs se aplicam também nessa situação. O manejo conservador, com o uso da nutrição parenteral, apresenta taxas de fechamento de $70 \%{ }^{27}$.

\section{Fístulas Hepatobiliares}

O extravasamento de bile a partir de pequenos ductos após trauma hepático é uma ocorrência comum. A apresentação clínica dessas fístulas, entretanto, é surpreenden- 
temente baixa, com taxas que variam de 1 a $5 \%$. Em um levantamento prospectivo de 482 pacientes com trauma hepático em que a drenagem biliar foi acuradamente documentada, essa se fez presente em $28 \%$ dos casos (cinco vezes a estatística relatada na literatura) ${ }^{28}$. Essas situações podem ser manejadas conservadoramente se há drenagem adequada. Embora tenha se reportado que coleções biliares peri-hepáticas estejam associadas com taxas elevadas de abscessos intra-abdominais, 80\% dessas são insignificantes e, portanto, com pouca possibilidade de causar morbi-mortalidade ${ }^{29}$.

As fístulas biliares, definidas como $50 \mathrm{ml}$ ou mais de secreção biliar persistindo por mais de duas semanas, têm taxas relatadas de 7 a $10 \%$ e ocorrem principalmente após lesões hepáticas classe três e quatro (Classificação da AAST - American Association for Surgery in Trauma). Nessas situações, a mínima quantidade de bile no campo deve determinar uma procura sistemática por algum ducto lesado. Os drenos de sucção fechada são essenciais em evitar seqüelas de coleções intra-abdominais ou intra-hepáticas não drenadas. É preferível uma fístula biliar controlada do que um bilioma ou peritonite biliar. Com drenagem adequada e sem obstrução distal, quase todas as fístulas biliares fecham espontaneamente. Na persistência de drenagem acima de 300 a 400ml, deve-se proceder à investigação e o exame mais simples é a fistulografia. Lacerações menores na presença de obstrução distal e grandes lacerações ductais, mesmo sem obstrução distal, provavelmente não fecharão espontaneamente e freqüentemente necessitarão de tratamento cirúrgico. A drenagem percutânea trans-hepática do ducto lacerado é uma manobra pré-operatória essencial, pois demonstra a necessidade de cirurgia emergencial e permite ao paciente se recuperar de outras lesões críticas. Adicionalmente, essa medida pode ser até terapêutica podendo o ducto lacerado cicatrizar sobre o stent. No pós-operatório permite a descompressão da reconstrução da anastomose biliodigestiva, preferencialmente uma hepatojejunostomia em Y de Roux ${ }^{30}$. A drenagem biliar controlada (colecistostomia ou coledocostomia com dreno em $\mathrm{T}$ ) pode ser efetiva em prevenir a formação de fístulas ductais, mas tem indicações limitadas e deve ser usada seletivamente ${ }^{29}$

Shahrudin descreve uma série de 175 pacientes com trauma hepático que necessitaram de tratamento cirúrgico. Os pacientes foram tratados com drenagem percutânea (nos biliomas) e todos tiveram boa evolução com tempo médio de fechamento de 44 dias ${ }^{31}$. Hollands descreve num estudo prospectivo de 306 pacientes, $13(04 \%)$ desenvolveram fístulas biliares e, com exceção de uma dessas, todas fecharam com o tratamento conservador num tempo médio de 33 dias ${ }^{32}$. Howdieshell relatou uma série de 175 pacientes com trauma hepático (lesões grau 3 e 4 da AAST) que necessitaram de hepatorrafia. Onze pacientes $(6 \%$ do total) desenvolveram complicações (um bilioma, duas fístulas biliares e oito casos com ambas) sendo seus diagnósticos entre o décimo quarto e o trigésimo dias de PO através de TC ou cintilografia. Todos obtiveram êxito com o manejo percutâneo ${ }^{33}$.

\section{Fístulas Pancreáticas}

Podem ser divididas em internas (ascite pancreática, derrame pleural pancreático) e externas (cutâneas ou com víscera oca) e ocorrem quando há lesão do ducto pancreático principal ou de um de seus ramos ${ }^{34}$. É a complicação mais frequiente após trauma duodenopancreático. Akhrass relatou uma série de 72 pacientes vítimas de trauma pancreático de etiologias múltiplas em que a ocorrência de fístula pancreática externa foi de $11 \%$ e mortalidade de 8 a $10 \%{ }^{35}$. Exteriorizam-se através de drenos, da ferida operatória ou drenagem percutânea de alguma coleção. Geralmente são percebidas entre o $2^{\circ}$ e o $7^{\circ}$ dias pós-trauma através de drenagem de secreção serosa com elevado nível de amilase $(1000-300000 \mathrm{u} / \mathrm{ml})^{6}$.

Tanto o trauma contuso como o penetrante estão associados à formação de fístulas e essas ocorrem em função de ressecção pancreática ou por falha houver detecção de lesão ductal. Cogbill relatou uma série de 74 pacientes submetidos a ressecções pancreáticas distais por trauma com uma incidência de fístula de $13,5 \%$ (dez casos) sendo que oito evoluíram com fechamento espontâneo ${ }^{36}$.

A TC é o método ideal para localizar coleções. Se essas forem sintomáticas, expansivas ou houver suspeita de infecção, devem ser submetidas à punção percutânea. A fistulografia pode ser útil para demonstrar a comunicação ductal e com outros órgãos. A colangiopancreatografia endoscópica retrógrada (CPER) é valiosa particularmente quando se planeja um tratamento cirúrgico. Sua utilidade baseia-se na definição de obstrução ductal, situação em que o fechamento espontâneo é menos provável.

O manejo terapêutico conservador é recomendado por quatro a seis semanas. Inclui drenagem livre e desimpedida da secreção, controle da sepse abdominal, suporte nutricional com nutrição parenteral, reposição das perdas e cuidados com a pele ${ }^{37}$. A utilização de bloqueadores dos receptores $\mathrm{H}_{2}$ ou da bomba de hidrogênio pode ser útil uma vez que diminuem o volume de secreção gástrica. Até $80 \%$ das fístulas pancreáticas fecham espontaneamente com essas medidas 38

Boden relatou os efeitos supressivos da somatostatina sobre a secreção pancreática ${ }^{39}$. $\mathrm{O}$ fator complicador seria sua meia-vida curta (menor de três minutos) o que obrigaria a sua administração contínua. Para tal foram desenvolvidos análogos como o octreotide que tem meia-vida de 2 horas e pode ser administrado via subcutânea. A dose inicial recomendada é de $50 \mathrm{mg}$, três vezes ao dia sendo ajustada de acordo com o débito podendo chegar ao máximo de $200 \mathrm{mg}$ três vezes ao dia. Os estudos demonstram que seu uso diminui o débito da fístula e o tempo para fechamento, embora não aumente o percentual de fístulas que fecham $4,38,40$.

Quando o manejo conservador falha, deve-se considerar a opção cirúrgica. Deve-se avaliar se o tratamento atual foi adequado, se há uma razão anatômica clara para a persistência da drenagem, se o estado nutricional foi corretamente avaliado e tratado e se o paciente está devidamente preparado para a cirurgia. O tipo de operação é ditado pela anatomia; o uso da TC e da CPER na defini- 
ção anatômica pré-operatória é crucial, pois identificam o nível de ressecção quando essa é indicada ${ }^{41}$. As fístulas de corpo e cauda sem estenose ductal devem ser submetidas à pancreatectomia distal. As lesões cefálicas, associadas ou não à estenose ductal ou a pseudocisto, devem ser submetidas à drenagem interna. A duodenopancreatectomia ou pancreatectomia subtotal (95\% do órgão) são raramente justificáveis em função de elevada morbimortalidade ${ }^{38}$.

\section{Fístulas Jejunoileais}

É o local de maior incidência de FDs, com alta incidência de desnutrição e sepse ( 25 a $75 \%)$, sendo essa a principal causa de morte. Sua etiologia inclui deiscência anastomótica ou lesões despercebidas. Deve-se suspeitar de pacientes cujo curso clínico é adequado e subitamente se altera com sepse associada. A ocorrência de peritonite difusa é comum e a mortalidade geral situa-se entre 15 e $25 \%$, enquanto as taxas de fechamento espontâneo variam de 40 a $70 \%$ 6,42,43.

As fístulas externas são de fácil reconhecimento. A presença de febre com ferida operatória eritematosa e drenagem de material purulento e/ou entérico é um quadro clássico que pode ser confirmado pela ingestão de algum corante (p.ex. azul de metileno) ou por fistulografia. A gravidade depende do sítio fistuloso e do débito. As fístulas internas são de mais difícil reconhecimento e requerem um alto grau de suspeição, pois podem ser menos sintomáticas ${ }^{42}$.

Os distúrbios hidreletrolíticos são comuns, ocorrendo em até $45 \%$ dos casos, devendo seu controle ser diário e sua reposição feita corretamente. A desnutrição está associada às fístulas de alto débito e pode ser exacerbada pela sepse. O uso da nutrição parenteral aumenta a taxa de fechamento espontâneo. A dieta enteral, quando factível, deve ser usada no suporte nutricional. Enquanto houver um foco infeccioso sem controle não haverá forma de reverter o catabolismo a que o paciente está exposto. Em pacientes estáveis, a TC pode ser útil na identificação de coleções a serem drenadas e o fistulograma pode fornecer dados sobre o sítio anatômico da lesão. O uso dos análogos da somatostatina está associado a uma redução do débito e aumento do índice de fechamento espontâneo. A maioria das séries indica que há pequena chance de fechamento se esse não ocorrer em quatro a seis semanas com o tratamento descrito acima, devendo então ser considerada a opção cirúrgica. A exploração deve ser indicada no paciente clinicamente instável, com irritação peritoneal difusa. O procedimento deve incluir drenagem de abscesso, lavagem ampla da cavidade e ressecção ou reparo da lesão se possível. Caso o paciente esteja séptico (com peritonite difusa) a exteriorização dos cotos para uma reconstrução futura é a opção mais segura ${ }^{42,43,44}$.

\section{Fístulas Colônicas}

Entre as FDs, as fístulas colônicas são as menos comuns. Freqüentemente são externas, de baixo débito, apresentam menos intercorrências e ocorrem principalmente devido a complicações pós-operatórias ${ }^{45}$. Sua incidência é de 1 a $2 \%$ nos reparos primários das lesões colônicas em geral ${ }^{46}$. Jacobson descreve uma série de 64 pacientes com lesões colônicas (48 por ferimentos por projétil de arma de fogo de baixa velocidade, sete ferimentos por projéteis de alta velocidade e nove ferimentos por arma branca) que foram tratados com reparo primário. Três pacientes foram reoperados em função de outras ocorrências e nenhum apresentou qualquer complicação relacionada aos reparos colônicos ${ }^{47}$.

O quadro clínico inclui febre, dor abdominal, massa palpável, sangramento retal, drenagem fecalóide pela ferida operatória e peritonite. A fistulografia, a TC e a retossigmoidoscopia podem ser úteis na avaliação dessas lesões. O uso do enema opaco deve ser seletivo em face dos riscos de agravar a perfuração, desfazendo o bloqueio e provocando peritonite grave. São descritas taxas de demonstração da fístula em até $90 \%$ dos casos ${ }^{45}$.

O manejo da sepse abdominal com drenagem percutânea é fundamental devido a grande contaminação associada. A infecção normalmente está localizada e ao alcance cirúrgico. A necessidade de um manejo mais agressivo depende da extensão do processo inflamatório, do débito e da dificuldade em controlar o efluente. Se o débito for baixo e não há abscesso associado, é grande a possibilidade de fechamento espontâneo, mesmo com dieta via oral. As lesões de alto débito fecham mais rapidamente com o uso da dieta parenteral e jejum. A diversão fecal proximal com drenagem do abscesso é útil se há uma grande coleção ou peritonite difusa e mais do que um pequeno defeito anastomótico. A ileostomia pode ser útil no controle proximal da secreção intestinal ${ }^{45}$.

\begin{abstract}
Digestive fistulas are associated with significant morbidity and mortality, representing mayor challenges regarding diagnosis and the requiring management according physiopathologic basis, including hydroeletrolytic therapy, antibiotics, nutritional support, selected surgical handling and skin care. Most of these lesions are postoperative complications, especially in urgency and traumatic situations. Our objective is to revise important aspects regarding gastrointestinal tract fistulas secondary to trauma, emphasizing classification, physiopathology, diagnosis, complications and treatment.
\end{abstract}

Key Words: Digestive fistula, Gastrintestinal tract; Trauma; Surgical complications. 


\section{REFERÊNCIA}

1. Berry SM, Fischer JE - Classification and pathophysiology of enterocutaneous fistulas. Surg Clin North Am, 1996, 76(5): 1009-18

2. Rolandelli R, Roslyn JJ - Surgical management and treatment of sepsis associated with gastrointestinal fistulas. Surg Clin North Am, 1996, 76(5): 1111-22

3. Campos ACL, Meguid MM, Coelho JCU - Factors influencing outcome in patients with gastrointestinal fistula. Surg Clin North Am, 1990, 76(5): 1191-98

4. Rubelowsky J, Machiedo GW - Reoperative versus conservative management for gastrointestinal fistulas. Surg Clin North Am, 1991, 71(1): 147-57

5. Prickett D, Montgomery R, Cheadle WG - External fistulas arising from the digestive tract. South Med J, 1991, 84(6):736-9

6. Foster CE, Lefor AT - General management of gastrointestinal fistulas. Surg Clin North Am, 1996, 76(5): 1019-33

7. Gerzof SG, Robbins AH, Johnson WC et al - Percutaneous catheter drainage of abdominal abscesses. N Engl J Med, 1981, 305(12): 653-7

8. Thomas HA - Radiologic investigation and treatment of gastrointestinal fistulas. Surg Clin North Am, 1996, 76(5): 1081-94

9. Dobrin PB, Gully PH, Greenle HB et al - Radiologic diagnosis of na intra-abdominal abscess. Arch Surg, 1986, 121:41-46

10. Haaga JR, Weinstein AJ - CT-guided percutaneous aspiration and drainage of abscesses. AJR, 1980, 135: 1187 94

11. Gerzof SG, Jonhson WC - Radiologic aspects of diagnosis and treatment of abdominal abscesses. Surg Clin North Am, 1984, 64:53-65

12. Fry DE, Osler T - Abdominal wall considerations and complications in reoperative surgery. Surg Clin North Am, 1991, 71:1-11

13. Chapman R, Foram R, Dunphy JE - Management of intestinal fistulas. Am J Surg, 1964, 108: 157-64

14. Meguid MM, Campos ACL - Nutritional management of patients with gastrointestinal fistulas. Surg Clin North Am, 1996, 76(5): 1035-80

15. Soeters PB, Ebeid AM, Fischer JE - Review of 404 patients with gastrointestinal fistulas. Ann Surg, 1979, 190: 189-202

16. Thomas RJ - The response of patients with fistulas of the gastrointestinal tract to parenteral nutrition. Surg Gynecol Obstet, 1981, 153: 77-80

17. Sitges-Serra A, Jaurrieta E, Sitges-Creus A - Management of postoperative enterocutaneous fistulas: the roles of parenteral nutrition and surgery. Br J Surg, 1982, 69: $147-50$

18. Meguid MM, Muscaritoli M - Current uses of total parenteral nutrition. Am Fam Physician, 1993, 47(2): 38394

19. Edmunds LH, Willians GM, Welch CE - External fistulas arising from the gastrointestinal tract. Ann Surg, 1960, $152: 445-71$

20. Symbas PN - "Injury to the esophagus, trachea, and bronchus". In Feliciano DV, Moore EE, Mattox KL (eds) - Trauma. $3^{\text {a }}$ Edição. Stanford. Editora Appleton \& Lange, 1996, pp:375-85
21. Han SY, Tishler JM - Perforation of the abdominal segment of the esophagus. AJR, 1984, 143: 751-4

22. Wilson RF - "Esophagus". In Maull KI, Rodriguez A, Wiles CE (eds) - Complications in trauma and critical care.Philadelphia. Editora WB Saunders, 1996, pp: 347-58

23. Sanchez-lloret J, Belda J, Canalis E - "Trauma de pared toracica, traquea, bronquios y esofago". In Rodriguez A, Ferrada R (eds) - Trauma, Sociedad Panamericana de Trauma. Colombia. 1997; 267-87

24. Shung MA, Wanebo HJ - Management and treatment of gastric and duodenal fistulas. Surg Clin North Am, 1996, 76(5): 1137-45

25. Jurkovich GJ - "Injury to the duodenum and pancreas". In Feliciano DV, Moore EE, Mattox KL (eds) - Trauma.

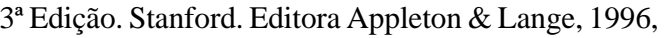
pp: 573-94

26. Asensio JA, Stewart BM, Demetriades D - "Duodenum". In Maull KI, Rodriguez A, Wiles CE (eds) - Complications in trauma and critical care. Philadelphia. Editora WB Saunders, 1996, pp: 364-79

27. Pearlstein L, Jones CE, Polk HC - Gastrocutaneous fistula: etiology and treatment. Ann Surg, 1978, 187: 223-6

28. Fabian TC et al. Factors affecting morbidity following hepatic trauma: a prospective analysis of 482 liver injuries. Ann Surg 1991, 213:540-7

29. Pachter HL, Nicastro JM - "Hepatic trauma". In Maull KI, Rodriguez A, Wiles CE (eds) - Complications in trauma and critical care. Philadelphia. Editora WB Saunders, 1996, pp:403-20

30. Pachter HL, Liang HG, Hofstetter SR - "Liver and biliary tract trauma". In Feliciano DV, Moore EE, Mattox KL (eds) - Trauma. $3^{\text {a }}$ Edição. Stanford. Editora Appleton \& Lange, 1996, pp:487-523

31. Sharundin MD, Noori SM - Biloma and biliary fistula associated with hepatorraphy for liver injury. Hepatogastroenterology, 1997, 44(14): 519-21

32. Hollands MJ, Little JM - Post-traumatic bile fistulae. J Trauma, 1991, 31(1): 117-20

33. Howdieshell TR, Purvis J, Bates WB et al - Biloma and biliary fistula following hepatorraphy for liver trauma: incidence, natural history, and management. Am Surg, 1995, 61(2): 165-8

34. Kozarek RA, Traverso LW - Pancreatic fistulas: etiology, consequences, and treatment. Gastroenterologist, 1996, 4(4):238-44

35. Akhrass R, Yaffe MB, Brandt CP et al - Pancreatic trauma: a ten-year multi-institutional experience. Am Surg, 1997, 63(7): 598-604

36. Cogbill TH, Moore EE, Morris JA Jr et al - Distal pancreatectomy for trauma: a multicenter experience. J Trauma, 1991, 31(12): 1600-6

37. Madiba TE, Haffejee AA, Singh B et al - Nutritional support in the management of external pancreatic fistulas. S Afr J Surg, 1995, 33(2): 81-4

38. Ridgeway MG, Stabile BE - Surgical management and treatment of pancreatic fistulas. Surg Clin North Am, 1996, 76(5): 1159-73

39. Boden G, Sivitz MC, Owen OE et al - Somatostatin suppresses secretion and pancreatic exocrine secretion. Science, 1975, 190: 163-5 
40. Barnes SM, Kontny BG, Prinz RA et al - Somatostatin ana$\log$ treatment of pancreatic fistulas. Int J Pancreatol, 1993, 14(2): 181-8

41. Stone A, Sugawa C, Lucas C et al - The role of endoscopic retrograde pancreatography (ERP) in blunt abdominal trauma. Am Surg, 1990, 56(11): 715-20

42. Tassiopoulos AK, Baum G, Halverson JD - Small bowel fistulas. Surg Clin North Am, 1996, 76(5): 1175-81

43. Wissner DH - "Injury to the stomach and small bowel". In Feliciano DV, Moore EE, Mattox KL (eds) - Trauma.

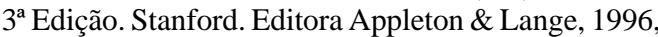
pp: 551-71

44. Stevens SL, Rubin BG - "Small Intestine". In Maull KI, Rodriguez A, Wiles CE (eds) - Complications in trauma and critical care. Philadelphia. Editora WB Saunders, 1996, pp:390-7

45. Lavery IC - Colonic fistulas. Surg Clin North Am, 1996, 76(5): 1183-90

46. Burch JM - "Injury to the colon and rectum". In Feliciano DV, Moore EE, Mattox KL (eds) - Trauma. $3^{\text {a Edi- }}$ ção. Stanford. Editora Appleton \& Lange, 1996, pp:595-613
47. Jacobson LE, Gomez GA, Broadie TA - Primary repair of 58 consecutive penetrating injuries of the colon: should colostomy be abandoned? Am Surg, 1997, 63(2): 170-7

Endereço para correspondência: Dr. Hamilton Petry de Souza

Rua Antonio Parreiras, 145/401

90450-050 — Porto Alegre-RS

E-mail:hpetry@conex.com.br 